\title{
A new integral representation for quasi-periodic scattering problems in two dimensions
}

\author{
Alex Barnett · Leslie Greengard
}

Received: 9 August 2010 / Accepted: 4 November 2010 / Published online: 23 November 2010

(C) The Author(s) 2010. This article is published with open access at Springerlink.com

\begin{abstract}
Boundary integral equations are an important class of methods for acoustic and electromagnetic scattering from periodic arrays of obstacles. For piecewise homogeneous materials, they discretize the interface alone and can achieve high order accuracy in complicated geometries. They also satisfy the radiation condition for the scattered field, avoiding the need for artificial boundary conditions on a truncated computational domain. By using the quasi-periodic Green's function, appropriate boundary conditions are automatically satisfied on the boundary of the unit cell. There are two drawbacks to this approach: (i) the quasi-periodic Green's function diverges for parameter families known as Wood's anomalies, even though the scattering problem remains well-posed, and (ii) the lattice sum representation of the quasi-periodic Green's function converges in a disc, becoming unwieldy when obstacles have high aspect ratio.

In this paper, we bypass both problems by means of a new integral representation that relies on the free-space Green's function alone, adding auxiliary layer potentials on the boundary of the unit cell strip while expanding the linear system to enforce quasi-periodicity. Summing nearby images directly leaves auxiliary densities that are smooth, hence easily represented in the Fourier domain using Sommerfeld integrals. Wood's anomalies are handled analytically by deformation of the Sommerfeld contour. The resulting integral equation is of the second kind and achieves spectral accuracy. Because of our image structure, inclusions which intersect the unit cell walls are handled easily and automatically. We include an implementation and simple code example with a freely-available MATLAB toolbox.
\end{abstract}

Communicated by Per Lötstedt.

A. Barnett

Department of Mathematics, Dartmouth College, Hanover, NH 03755, USA

e-mail: ahb@math.dartmouth.edu

L. Greengard $(\bowtie)$

Courant Institute, New York University, 251 Mercer St, New York, NY 10012, USA

e-mail: greengard@cims.nyu.edu 
Mathematics Subject Classification (2000) 35J25 - 65R20 - 65N38 - 78A45 • $35 \mathrm{P} 25$

\section{Introduction and problem formulation}

A number of problems in wave propagation require the calculation of quasi-periodic solutions to the Helmholtz or Maxwell equations in the frequency domain. A typical example in two dimensions involves computing the field scattered by an infinite onedimensional array (a grating) of dielectric obstacles in response to an incoming plane wave (Fig. 1) [38].

For the sake of simplicity, we will restrict our attention here to the analysis of the two-dimensional (locally isotropic) Maxwell equations in what is usually called TM polarization $^{1}[18,19]$. In this case, the full Maxwell equations for the electric and magnetic fields $\mathbf{E}, \mathbf{H}$,

$$
\begin{gathered}
\nabla \times \mathbf{E}=i \omega \mu \mathbf{H}, \\
\nabla \times \mathbf{H}=-i \omega \epsilon \mathbf{E},
\end{gathered}
$$

take the simpler form

$$
\begin{aligned}
& \mathbf{E}(x, y, z)=\mathbf{E}(x, y)=\left(0,0, u^{\mathrm{t}}(x, y)\right), \\
& \mathbf{H}(x, y, z)=\mathbf{H}(x, y)=\frac{1}{i \omega \mu}\left(u_{y}^{\mathrm{t}}(x, y),-u_{x}^{\mathrm{t}}(x, y), 0\right),
\end{aligned}
$$

where $u^{\mathrm{t}}$ is a scalar field defined in $\mathbb{R}^{2}$. Here, $\epsilon$ and $\mu$ are the permittivity and permeability, respectively, and we have assumed a time dependence of $e^{-i \omega t}$ at frequency $\omega>0$. The index of refraction $n=n(x, y)$ is defined to be $n=\sqrt{\epsilon \mu}$. We will concentrate on materials with piecewise constant index of refraction, and assume that units are scaled so that $n=1$ in the background material and $n \neq 1$ in the obstacles themselves (Fig. 1). In order to satisfy the full Maxwell equations, it is straightforward to verify that $u^{\mathrm{t}}$ must satisfy the scalar Helmholtz equations

$$
\begin{aligned}
\left(\Delta+n^{2} \omega^{2}\right) u^{\mathrm{t}}=0 & \text { in } \Omega_{\Lambda}, \\
\left(\Delta+\omega^{2}\right) u^{\mathrm{t}}=0 & \text { in } \mathbb{R}^{2} \backslash \overline{\Omega_{\Lambda}} .
\end{aligned}
$$

Imposing the standard continuity conditions on the tangential components of the electric and magnetic fields [18, 19], we have

$$
u^{\mathrm{t}^{+}}-u^{\mathrm{t}-}=u_{n}^{\mathrm{t}+}-u_{n}^{\mathrm{t}-}=0 \quad \text { on } \partial \Omega_{\Lambda},
$$

where the superscripts $+(-)$ denote the limiting values on the surface approaching from outside (inside), and $u_{n}^{\mathrm{t}}:=\partial u^{\mathrm{t}} / \partial n$ is the outward-pointing normal derivative. Writing $\mathbf{x}=(x, y)$, the incoming plane wave with angle $\theta$ takes the form

\footnotetext{
${ }^{1}$ The reader should be warned that in this setting some authors [5] use TM and TE in the reversed sense.
} 

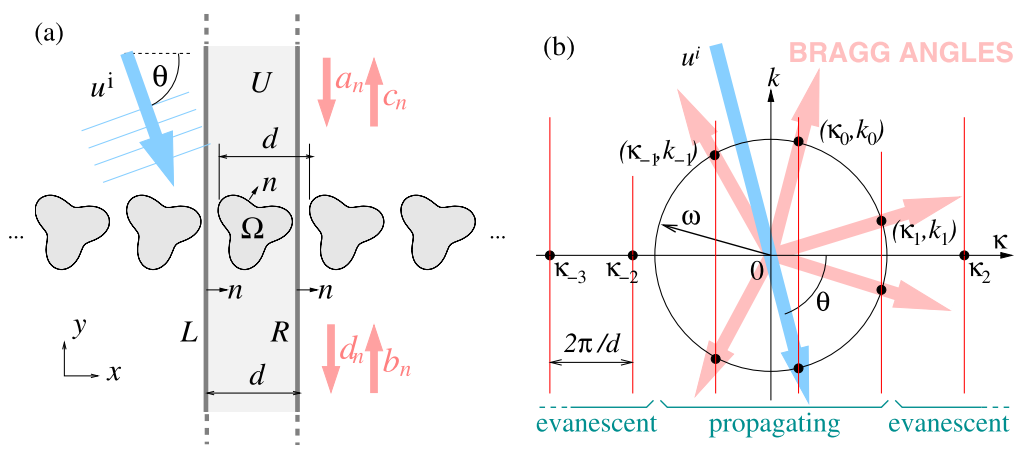

Fig. 1 (a) A simple 2D grating scattering geometry: a plane wave incident on a one-dimensional array of obstacles with periodicity $d . \theta \in(-\pi, 0)$ is the angle of incidence with respect to the $x$-axis. $\Omega \subset \mathbb{R}^{2}$ denotes the single obstacle lying in the "unit cell" $U:=(-d / 2, d / 2) \times(-\infty, \infty)$. $\Omega_{\Lambda}$ denotes the set of all obstacles in the array, and $\mathbb{R}^{2} \backslash \overline{\Omega_{\Lambda}}$ denotes the background material. (b) Fourier (k-space) picture showing construction of Rayleigh-Bloch wavevectors, the propagating ones of which give the angles of Bragg diffraction orders

$$
u^{\mathrm{i}}(\mathbf{x})= \begin{cases}0 & \text { in } \Omega_{\Lambda}, \\ e^{i \mathbf{k} \cdot \mathbf{x}} & \text { in } \mathbb{R}^{2} \backslash \overline{\Omega_{\Lambda}},\end{cases}
$$

where the wavenumber is $\mathbf{k}=\left(\kappa^{\mathrm{i}}, k^{\mathrm{i}}\right):=(\omega \cos \theta, \omega \sin \theta)$. Since $|\mathbf{k}|=\omega, u^{\mathrm{i}}$ satisfies the appropriate Helmholtz equation in the background material. We will make frequent use of the following.

Definition 1 A function $f: \mathbb{R}^{2} \rightarrow \mathbb{C}$ is said to be quasi-periodic ${ }^{2}$ with phase $\alpha$, $|\alpha|=1$, if

$$
f(x+d, y)=\alpha f(x, y) \quad \forall(x, y) \in \mathbb{R}^{2} .
$$

We interpret $\alpha$ as the Bloch phase associated with translation by one period. In particular, the incident wave is quasi-periodic with phase

$$
\alpha=e^{i \kappa^{\mathrm{i}} d}=e^{i \omega d \cos \theta} .
$$

(This condition reduces to true periodicity only when $\omega d \cos \theta=2 n \pi$ for some integer $n$, such as when the incoming wave is normally incident, i.e. $\theta=-\pi / 2$.) By the translational symmetry of the problem, the resulting total field $u^{\mathrm{t}}$ must be similarly quasi-periodic.

In the language of scattering theory, we may now write the total field as $u^{\mathrm{t}}=$ $u^{\mathrm{i}}+u$, where $u$ is the scattered field. It is easy to see that $u$ satisfies the homogeneous Helmholtz equations (1.1)-(1.2), and that (1.3) results in the inhomogeneous interface conditions

$$
u^{+}-u^{-}=-u^{\mathrm{i}}, \quad u_{n}^{+}-u_{n}^{-}=-u_{n}^{\mathrm{i}} \quad \text { on } \partial \Omega_{\Lambda} .
$$

\footnotetext{
${ }^{2}$ Also known as pseudoperiodic in some literature.
} 
Moreover, $u$ is quasi-periodic.

Finally, $u$ satisfies the following radiation condition [9]. Let $y_{0}>0$ be sufficiently large that the rectangle $(-d / 2, d / 2) \times\left(-y_{0}, y_{0}\right)$ contains $\bar{\Omega}$. Define $\kappa_{n}=$ $\kappa^{\mathrm{i}}+2 \pi n / d$, and $k_{n}=+\sqrt{\omega^{2}-\kappa_{n}^{2}}$ where the sign is taken as positive real or positive imaginary. Then the plane wave with wavevector $\left(\kappa_{n}, k_{n}\right)$ is quasi-periodic for each integer $n$, and satisfies the Helmholtz equation at frequency $\omega$; a geometric construction for these wavevectors is given in Fig. 1(b). The radiation condition on $u$ is that it has uniformly-convergent upwards and downwards Rayleigh-Bloch expansions

$$
\begin{aligned}
& u(x, y)=\sum_{n \in \mathbb{Z}} c_{n} e^{i \kappa_{n} x} e^{i k_{n}\left(y-y_{0}\right)} \quad y>y_{0}, x \in \mathbb{R}, \\
& u(x, y)=\sum_{n \in \mathbb{Z}} d_{n} e^{i \kappa_{n} x} e^{i k_{n}\left(-y-y_{0}\right)} \quad y<-y_{0}, x \in \mathbb{R} .
\end{aligned}
$$

The complex coefficients $c_{n}, d_{n}$, for $n$ such that $\left|\kappa_{n}\right| \leq \omega$ (propagating waves), are the Bragg diffraction amplitudes at the grating orders. For all other $n$ these give evanescent components which do not contribute to the far field.

The above conditions result in the existence of a solution $u$ for all frequencies $\omega$ and incident angles $\theta$ [9, Thm. 3.2]. The solution is unique (i.e. the problem wellposed) at all but a discrete set of frequencies $\omega$, for each fixed horizontal wavenumber $\kappa^{\mathrm{i}}=\omega \cos \theta$ [9, Thm. 3.4]. These discrete frequencies correspond to guided modes, which propagate without loss along the grating. The only such modes relevant in the scattering setting are nonrobust in the sense that they are embedded in the continuous spectrum, and therefore disappear under generic perturbations [40]. Such modes occur at isolated points in $\left(\omega, \kappa^{\mathrm{i}}\right)$ parameter space, and may cause anomalous transmission through the grating [41]. (There may also exist robust guided modes with frequencies below the continuous spectrum corresponding to the mode's horizontal wavenumber, which are therefore inaccessible in the scattering setting.) If $n<1$ and $\Omega$ is shaped such that the refractive index is non-decreasing with $|y|$ then no guided modes can exist [9, Thm. 3.5]. Conversely, for arbitrary $\Omega$ and $n>1$, at least one guided mode must exist [9, Thm. 4.4(2)] (although the existence of nonrobust modes is not guaranteed). The above is carefully reviewed (in the three-dimensional setting) by Shipman [40].

There is another entirely different type of "resonance" that may occur, first observed experimentally by Wood [46]: there are parameters where the diffraction intensities may change arbitrarily rapidly with respect to $\omega$ or $\theta$. These so-called Wood's anomalies are now known to occur when, for some $n, \kappa_{n}= \pm \omega$ thus $k_{n}=0$ and the $n$th Rayleigh-Bloch mode in (1.6)-(1.7) is constant in $y$ (a horizontally traveling plane wave). It is also possible to have a double Wood's anomaly, when $\kappa_{n}=-\kappa_{m}=\omega$ for a pair of integers $n, m$; clearly this can only occur for integer $\omega d / 2 \pi$. A thorough analysis of Wood's anomalies in the case of disc obstacles is given by Linton-Thompson [27]. We emphasize that, unlike guided modes discussed above, Wood's anomalies do not prevent the boundary value problem for $u$ from having a unique solution. One of the key features of the method we present is that it works at Wood's anomalies. 
Applications of the above diffraction problem are widespread, including gratings, antennae, acoustic diffusers, and small-amplitude water waves [37]. Generalizing slightly to more than one inclusion per unit cell, one is able to study finitethickness photonic crystal slabs [41, 43], with applications to integrated optics and signal processing. Moreover, we expect the tools we present here to generalize well to a much larger class of technologically-important scattering problems, including periodic layered media with inclusions. Modern applications include dielectric gratings [8], process control in semiconductor lithography [30], efficient thin-film photovoltaic cells [4, 20], and integrated nano-scale optical devices [19]. Some of these applications (e.g. photonic crystals, silicon microwire arrays [20]) demand structures that are many periods deep, i.e. high aspect ratio.

A variety of existing numerical methods have been applied to periodic scattering problems. Unfortunately, many of the popular methods are only low order accurate and agree in test problems to only 1 digit of accuracy [45]. Finite difference approximation to the time-domain wave equation (FDTD) [16, 42] is common; it has the advantage of being quite general, but has the disadvantage of requiring large regions of free space to be simulated, with significant dispersion error for electrically large domains. FDTD methods also require non-reflecting boundary conditions and long settling times for resonant structures. Moving to the frequency domain, finite element methods $[5,30]$ require non-trivial grid generation, especially in three dimensions, and also demand the discretization of substantial regions of free space together with the imposition of non-local radiation conditions constructed from (1.6) and (1.7). The engineering community has developed many other methods-including coupled wave analysis, the waveguide method [45], the C method [24], and cylindrical (multipole) expansions [27, 37]—which are often based on uncontrolled approximations.

In this work we use integral equations (sometimes known as boundary element methods). These have a rigorous mathematical foundation [11, 34], and have the advantages of discretizing the material interfaces alone, of automatically enforcing radiation conditions, and of high-order convergence, which allows high accuracies to be reached very efficiently. The standard way to adapt integral equations to solve periodic scattering problems is by replacing the free-space kernel (see (2.7)) by the quasi-periodic Green's function (the kernel appearing in (3.4) with $P=\infty$ ); see [35, $36,43]$. Considerable effort has been spent on the tricky task of computing this quasiperiodic Green's function efficiently (see, for example, [3, 12, 25, 28, 31]). The state of the art appears to be around $10^{-4}$ to $10^{-3} \mathrm{sec}$ per evaluation at low frequencies [3], and $10^{-1} \mathrm{sec}$ at very high frequencies [23].

A much more serious problem is that the quasi-periodic Green's function blows up at Wood's anomalies with a $-1 / 2$ power singularity [25, Eq. (2.13)-(2.14)], causing any method using it to have divergent round-off error near to, and failure at, each such anomaly. In the related case of periodic surface scattering, Zhang and Chandler-Wilde [47] modified the Green's function to that of a half-space, which cures this divergence (this was implemented in [2]); however, this idea fails to help in our case of disconnected obstacles. A final problem is that the quasi-periodic Green's function is often computed using lattice sums [25, 26], which is natural when using fast multipole acceleration in large-scale scattering problems [36]. However, since this representation converges in discs (or spheres in 3D), it becomes cumbersome for high aspect ratio 
geometries. We believe that the above reasons highlight the need for the more robust and flexible scheme that we present here.

We propose a periodizing scheme which builds upon the single-obstacle scattering problem without changing the kernel, allowing existing code (quadrature, fast multipole schemes, etc.) to be used unaltered. Our scheme has much in common with that of [7], except that we only have one dimension of periodicity, requiring Fourier methods to handle the unbounded nature of the other dimension.

We review the non-periodic scattering scheme in Sect. 2, and present our new method in Sect. 3. A simple numerical example, and then a high-aspect ratio one, are demonstrated in Sect. 4, where we also compute Bragg diffraction amplitudes $c_{n}$ and $d_{n}$ from (1.6)-(1.7). Slight modifications of the method allow handling of Wood's anomalies in Sect. 5 and obstacles which touch (wrap around) the unit cell walls in Sect. 6. We conclude in Sect. 7, and give a short code example in the Appendix.

\section{Transmission scattering from a single obstacle}

The approach of this paper builds directly upon the boundary integral method for the scattering problem for a single isolated obstacle $\Omega$, whose formulation and solution we now review. Given the incoming wave $u^{\mathrm{i}}$ given by (1.4), one seeks the scattered wave $u$ satisfying the Helmholtz equations

$$
\begin{aligned}
\left(\Delta+n^{2} \omega^{2}\right) u=0 & \text { in } \Omega, \\
\left(\Delta+\omega^{2}\right) u=0 & \text { in } \mathbb{R}^{2} \backslash \bar{\Omega},
\end{aligned}
$$

the inhomogeneous boundary conditions (1.5) on the single boundary $\partial \Omega$, and the free space Sommerfeld radiation condition [10]

$$
\frac{\partial u}{\partial r}-i \omega u=o\left(r^{-1 / 2}\right), \quad r:=|\mathbf{x}| \rightarrow \infty .
$$

To formulate this as a system of integral equations, we choose the standard RokhlinMüller scheme [33, 39],

$$
u= \begin{cases}\mathcal{S}^{(n \omega)} \sigma+\mathcal{D}^{(n \omega)} \tau & \text { in } \Omega, \\ \mathcal{S}^{(\omega)} \sigma+\mathcal{D}^{(\omega)} \tau & \text { in } \mathbb{R}^{2} \backslash \bar{\Omega},\end{cases}
$$

where the usual single and double layer densities [10] at any wavenumber $\omega>0$ are defined by

$$
\begin{aligned}
& \left(\mathcal{S}^{(\omega)} \sigma\right)(\mathbf{x})=\int_{\partial \Omega} G^{(\omega)}(\mathbf{x}, \mathbf{y}) \sigma(\mathbf{y}) d s_{\mathbf{y}} \\
& \left(\mathcal{D}^{(\omega)} \tau\right)(\mathbf{x})=\int_{\partial \Omega} \frac{\partial G^{(\omega)}}{\partial n_{\mathbf{y}}}(\mathbf{x}, \mathbf{y}) \tau(\mathbf{y}) d s_{\mathbf{y}} .
\end{aligned}
$$

Here the kernel $G^{(\omega)}(\mathbf{x}, \mathbf{y}):=G^{(\omega)}(\mathbf{x}-\mathbf{y})$ is the free-space Green's function,

$$
G^{(\omega)}(\mathbf{x})=\frac{i}{4} H_{0}^{(1)}(\omega|\mathbf{x}|), \quad \mathbf{x} \in \mathbb{R}^{2} \backslash\{\mathbf{0}\},
$$


where $H_{0}^{(1)}$ is the outgoing Hankel function of order zero.

The representation (2.4) already satisfies (2.1)-(2.2) and (2.3). Taking limits on the surface, using the standard jump relations for single and double layer potentials $[10,14]$, we can evaluate the mismatch (boundary inhomogeneity),

$$
\begin{aligned}
{\left[\begin{array}{c}
u^{+}-u^{-} \\
u_{n}^{+}-u_{n}^{-}
\end{array}\right] } & =\left(\left[\begin{array}{ll}
I & 0 \\
0 & I
\end{array}\right]+\left[\begin{array}{cc}
D^{(\omega)}-D^{(n \omega)} & S^{(n \omega)}-S^{(\omega)} \\
T^{(\omega)}-T^{(n \omega)} & D^{(n \omega) *}-D^{(\omega) *}
\end{array}\right]\right)\left[\begin{array}{c}
\tau \\
-\sigma
\end{array}\right] \\
& =: A_{0} \eta .
\end{aligned}
$$

Here $I$ is the identity operator, while $S$ and $D$ are defined to be the limiting boundary integral operators (maps from $C(\partial \Omega) \rightarrow C(\partial \Omega)$ ) with the kernels $\mathcal{S}$ and $\mathcal{D}$ interpreted in the principal value sense, and $D^{*}$ is the adjoint of $D$. ( $S$ is actually weakly singular so the limit is already well defined. A standard calculation $[10,14]$ shows that $D$ is weakly singular as well). The hypersingular operator $T$ has the kernel $\frac{\partial^{2} G(\mathbf{x}, \mathbf{y})}{\partial n_{\mathbf{x}} \partial n_{\mathbf{y}}}$ and is unbounded as a map from $C(\partial \Omega) \rightarrow C(\partial \Omega)$. In these definitions, as in (2.5)(2.6), it is implied that $G$ inherits the appropriate superscripts from $S, D$ and $T$. Equating (2.8) with the known mismatch (1.5) gives the following linear equation for the unknown densities $\eta:=[\tau ;-\sigma]$,

$$
A_{0} \eta=\left[\begin{array}{l}
-\left.u^{\mathrm{i}}\right|_{\partial \Omega} \\
-\left.u_{n}^{\mathrm{i}}\right|_{\partial \Omega}
\end{array}\right]=: b .
$$

Because only the difference of hypersingular $T$ operators appears in (2.8), $A_{0}$ is a compact perturbation of the identity, and (2.9) is a Fredholm system of integral equations of the second kind. Furthermore, it has a unique solution at all $\omega[10$, Thm. 3.41].

We apply the Nyström method to discretize (2.9) with $N$ quadrature points $\left\{\mathbf{y}_{j}\right\}_{j=1}^{N}$ on $\partial \Omega$, using the periodic trapezoid rule in the curve parametrization, corrected by the Martensen-Kussmaul weight scheme to handle the weak diagonal singularities, as proposed by Kress [21] (also see [7, Sect. 3.1]). This approximates (2.9) by the $2 N$-by- $2 N$ linear system

$$
\boldsymbol{A}_{0} \boldsymbol{\eta}=\boldsymbol{b}
$$

where $\eta$ is the length- $2 N$ column vector of approximations to the densities $[\tau ;-\sigma]$ sampled at the quadrature points, and $\boldsymbol{b}$ the similar vector of samples of the righthand side of (2.9). This scheme gives spectral accuracy, that is, the sampled densities tend to their exact values with error $O\left(e^{-c N}\right)$ for some $c>0$.

\section{Quasi-periodizing scheme}

We restrict attention to the single unbounded strip "unit cell" $U:=\{(x, y) \in$ $(-d / 2, d / 2) \times(-\infty, \infty)\}$, in which we can reformulate the boundary value problem for $u$ (as in, e.g. [9]). Recall that in $U, u$ satisfies the homogeneous partial differential equations (2.1)-(2.2) with inhomogeneous boundary conditions (1.5) on $\partial \Omega$. We introduce the discrepancy functions

$$
f:=\left.u\right|_{L}-\left.\alpha^{-1} u\right|_{R},
$$




$$
f_{n}:=\left.u_{n}\right|_{L}-\left.\alpha^{-1} u_{n}\right|_{R},
$$

which measure the failure of the scattered field to satisfy quasi-periodicity between the left $(L)$ and right $(R)$ walls of $U$. Note that the normals on both walls are defined in the positive $x$-direction (see Fig. 1(a)). Quasi-periodicity implies vanishing discrepancy,

$$
f(y)=f_{n}(y)=0, \quad y \in \mathbb{R} .
$$

Moreover, by the unique continuation of Cauchy data for the Helmholtz equation [13], the converse holds. Thus the above conditions on $u$, combined with the radiation conditions (1.6)-(1.7), are equivalent to that of Sect. 1. We may interpret this as a twoport "channel" or waveguide scattering problem in $U$, with the radiation conditions being that $u$ has no incoming channel modes from either above or below.

\subsection{Scattered field representation and linear system in spatial domain}

Our representation builds on (2.4). We first include a direct sum of neighboring phased copies of the obstacle which, as we shall see, will make the subsequent correction to enforce quasi-periodicity very simple. For this, we define

$$
\begin{aligned}
& \left(\tilde{\mathcal{S}}^{(\omega)} \sigma\right)(\mathbf{x})=\int_{\partial \Omega} \sum_{j=-P}^{P} \alpha^{j} G^{(\omega)}(\mathbf{x}, \mathbf{y}+j \mathbf{d}) \sigma(\mathbf{y}) d s_{\mathbf{y}}, \\
& \left(\tilde{\mathcal{D}}^{(\omega)} \tau\right)(\mathbf{x})=\int_{\partial \Omega} \sum_{j=-P}^{P} \alpha^{j} \frac{\partial G^{(\omega)}}{\partial n_{\mathbf{y}}}(\mathbf{x}, \mathbf{y}+j \mathbf{d}) \tau(\mathbf{y}) d s_{\mathbf{y}},
\end{aligned}
$$

where $\mathbf{d}=(d, 0)$ is the lattice translation vector. $P=0,1, \ldots$ is a numerical parameter that we may adjust to improve the convergence rate of the scheme. We add auxiliary densities on the unit cell walls as follows. We use the notation $\mathcal{S}_{W}$ and $\mathcal{D}_{W}$ to denote the analogs of (2.5)-(2.6) on the curve $W$, where $W$ is one of the two walls $L$ or $R=L+\mathbf{d}$. We represent the scattered field in $U$ by

$$
u= \begin{cases}\mathcal{S}^{(n \omega)} \sigma+\mathcal{D}^{(n \omega)} \tau & \text { in } \Omega, \\ \tilde{\mathcal{S}}^{(\omega)} \sigma+\tilde{\mathcal{D}}^{(\omega)} \tau+\left(\mathcal{S}_{L}+\alpha \mathcal{S}_{R}\right) \mu+\left(\mathcal{D}_{L}+\alpha \mathcal{D}_{R}\right) v & \text { in } U \backslash \bar{\Omega}\end{cases}
$$

where $\mu$ and $\nu$ are unknown single- and double-layer density functions on the real line $\{y \in \mathbb{R}\}$. We will stack these densities into the column vector $\xi:=[\nu ;-\mu]$.

Imposing the mismatch condition (1.5), and also the new quasi-periodicity condition (3.3) in the form $\left[f ; f_{n}\right]=0$, gives the two block rows of a linear operator system

$$
E\left[\begin{array}{l}
\eta \\
\xi
\end{array}\right]:=\left[\begin{array}{ll}
A & B \\
C & Q
\end{array}\right]\left[\begin{array}{l}
\eta \\
\xi
\end{array}\right]=\left[\begin{array}{l}
b \\
0
\end{array}\right],
$$

where $b$ is defined as before by (2.9). Notice that the lower part of the right-hand side vector is zero because the incident wave contributes no discrepancy. 
(a)

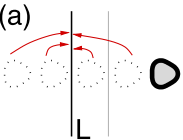

(b)

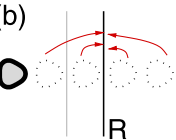

(c)

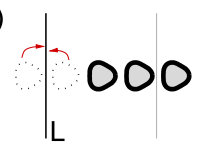

(d)

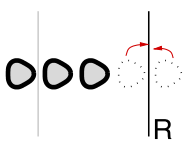

Fig. 2 Cancellations in discrepancy occurring in $C$ operator block, for the case $P=2$. Each arrow represents the influence of a source density on a target segment. For $Z=0$ (standard method), the effect of the four source images (dotted) on $L$ in (a) cancel the effect of the four source images on $R$ in (b). For $Z=1$ (which handles intersection of $\partial \Omega$ with unit cell walls) shown in (c) and (d), now only two source images cancel. See Sect. 6 for a discussion of the parameter $Z$

The four operators in the $2 \times 2$ blocks of $E$ capture the various effects of the obstacle and auxiliary densities on the mismatch on $\partial \Omega$ and the discrepancy. In order to describe them, we will need notation for the free-space boundary integral operators from a source curve $W$ to a (possibly distinct) target curve $V$,

$$
\begin{array}{ll}
\left(S_{V, W} \sigma\right)(\mathbf{x})=\int_{W} G^{(\omega)}(\mathbf{x}, \mathbf{y}) \sigma(\mathbf{y}) d s_{\mathbf{y}} & \mathbf{x} \in V, \\
\left(D_{V, W} \tau\right)(\mathbf{x})=\int_{W} \frac{\partial G^{(\omega)}}{\partial n_{\mathbf{y}}}(\mathbf{x}, \mathbf{y}) \tau(\mathbf{y}) d s_{\mathbf{y}} & \mathbf{x} \in V, \\
\left(D_{V, W}^{*} \sigma\right)(\mathbf{x})=\int_{W} \frac{\partial G^{(\omega)}}{\partial n_{\mathbf{x}}}(\mathbf{x}, \mathbf{y}) \sigma(\mathbf{y}) d s_{\mathbf{y}} & \mathbf{x} \in V, \\
\left(T_{V, W} \tau\right)(\mathbf{x})=\int_{W} \frac{\partial^{2} G^{(\omega)}}{\partial n_{\mathbf{x}} \partial n_{\mathbf{y}}}(\mathbf{x}, \mathbf{y}) \tau(\mathbf{y}) d s_{\mathbf{y}} \quad \mathbf{x} \in V .
\end{array}
$$

When $V=W$, these operators are to be understood in the principal value sense. When neither $V$ nor $W$ is given, they are both assumed to be $\partial \Omega$, as in Sect. 2. By analogy with (3.4), (3.5), versions of these operators whose kernels include the phased sum over $2 P+1$ images of the source curve will be indicated with a tilde $(\sim)$. Note that * no longer signifies operator adjoint, since the adjoint of $D_{V, W}$ is $D_{W, V}^{*}$.

We now give formulae for the four operator blocks in $E$. By analogy with (2.8), (3.6) gives

$$
A=\left[\begin{array}{ll}
I & 0 \\
0 & I
\end{array}\right]+\left[\begin{array}{cc}
\tilde{D}-D^{(n \omega)} & S^{(n \omega)}-\tilde{S} \\
\tilde{T}-T^{(n \omega)} & D^{(n \omega) *}-\tilde{D}^{*}
\end{array}\right] .
$$

Since the $j \neq 0$ terms here involve only non-local interactions, $A$ remains 2nd kind for $P>0$. The $B$ block describes the auxiliary densities' effect on the mismatch, and follows easily from (3.6),

$$
B=\left[\begin{array}{cc}
D_{\partial \Omega, L} & -S_{\partial \Omega, L} \\
T_{\partial \Omega, L} & -D_{\partial \Omega, L}^{*}
\end{array}\right]+\alpha\left[\begin{array}{cc}
D_{\partial \Omega, R} & -S_{\partial \Omega, R} \\
T_{\partial \Omega, R} & -D_{\partial \Omega, R}^{*}
\end{array}\right] .
$$

The $C$ block describes the obstacle densities' effect on the discrepancy functions $\left[f ; f_{n}\right]$ of (3.1)-(3.2). By translational symmetry, as shown in Fig. 2, there are can- 
cellations of $2 P$ terms in each subblock of $C$, leaving only

$$
\begin{aligned}
C= & \alpha^{P}\left[\begin{array}{ll}
D_{L-P \mathbf{d}, \partial \Omega} & -S_{L-P \mathbf{d}, \partial \Omega} \\
T_{L-P \mathbf{d}, \partial \Omega} & -D_{L-P \mathbf{d}, \partial \Omega}^{*}
\end{array}\right] \\
& -\alpha^{-P-1}\left[\begin{array}{ll}
D_{L+(P+1) \mathbf{d}, \partial \Omega} & -S_{L+(P+1) \mathbf{d}, \partial \Omega} \\
T_{L+(P+1) \mathbf{d}, \partial \Omega} & -D_{L+(P+1) \mathbf{d}, \partial \Omega}^{*}
\end{array}\right] .
\end{aligned}
$$

The result is that $C$ only involves interactions of distance at least $P d$ (this is similar to cancellation in [7]). Thus, increasing $P$ can improve the convergence rate of the quadrature scheme for the solution of the full system (3.7).

Finally, the $Q$ block gives the effect of auxiliary densities on the wall discrepancy itself. Inserting the $\mu$ and $v$ terms of (3.6) into (3.1)-(3.2) one easily gets,

$$
Q=\left[\begin{array}{ll}
I & 0 \\
0 & I
\end{array}\right]+\alpha\left[\begin{array}{cc}
D_{L, R} & -S_{L, R} \\
T_{L, R} & -D_{L, R}^{*}
\end{array}\right]-\alpha^{-1}\left[\begin{array}{cc}
D_{R, L} & -S_{R, L} \\
T_{R, L} & -D_{R, L}^{*}
\end{array}\right] .
$$

Now the reason becomes clear for choosing in (3.6) densities on the right wall which are suitably phased copies of those on the left: cancellation of the wall $L, L$ and $R, R$ self-interaction terms occurred in the above evaluation of $Q$, as shown in Fig. 2(c), (d). This means that $Q$ is the identity plus a convolution operator involving interactions of distance at least $d$, hence that the linear integral equation system (3.7) is of the 2nd kind. Note that, if densities were placed on $L$ alone, by contrast, no cancellation would occur, and the $L, L$ self-interaction would prevent (3.7) from being 2nd kind. Because the walls $L$ and $R$ are infinitely long, (3.7) is not usable in practice; however, we will presently transform it into a useful form. (We omit a rigorous analysis of solvability. That requires some additional work, since $E$ acts on functions some of which live on unbounded domains and is not a compact perturbation of the identity.)

\subsection{Fourier representation of layer potentials and discrepancy}

The wall densities $\mu, v$ in a solution of (3.7) represent the field due to the remainder of the infinite line of obstacle densities not accounted for by the direct sum in (3.6), namely the obstacles labelled $\ldots, P-2, P-1$ and $P+1, P+2, \ldots$ Since they must represent Rayleigh-Bloch modes (1.6)-(1.7), these densities generally do not decay as $|y| \rightarrow \infty$. Therefore any attempt to spatially truncate densities on $L$ and $R$ would give very slow convergence. To avoid this, we will work instead with the Fourier transform in the $y$ direction, using the usual definitions

$$
\hat{g}(k)=\frac{1}{2 \pi} \int_{-\infty}^{\infty} e^{-i k y} g(y) d y, \quad g(y)=\int_{-\infty}^{\infty} e^{i k y} \hat{g}(k) d k,
$$

where, as before, $k$ denotes the $y$-wavenumber. We will also need the Cartesian spectral representation for the free space Green's function [32, Ch. 7.2],

$$
G^{(\omega)}(\mathbf{x})=\frac{i}{4 \pi} \int_{-\infty}^{\infty} e^{i k y} \frac{e^{i \sqrt{\omega^{2}-k^{2}}|x|}}{\sqrt{\omega^{2}-k^{2}}} d k, \quad \mathbf{x}=(x, y) .
$$


Here the square-roots are taken positive real or positive imaginary, which may be achieved by taking the branch cuts of the function $\sqrt{\omega^{2}-k^{2}}$ in the $k$ plane as $(-\infty,-\omega) \cup(\omega,+\infty)$ along the real axis, and a Sommerfeld contour passing from the 2nd to 4th quadrants (see Fig. 3). Examining (3.16), we see that horizontal separation $(|x|>0)$ leads to exponentially decaying tails in the Fourier representation, which we will be able to truncate with spectral accuracy.

We define "Fourier layer potentials" by inserting (3.15) and (3.16) into the definition of layer potentials living on a vertical wall $W=\left\{\left(x_{0}, y\right): y \in \mathbb{R}\right\}$,

$$
\begin{aligned}
\left(\hat{\mathcal{S}}_{W} \hat{\mu}\right)(\mathbf{x}) & =\frac{i}{2} \int_{-\infty}^{\infty} e^{i k y} e^{i \sqrt{\omega^{2}-k^{2}}\left|x-x_{0}\right|} \frac{1}{\sqrt{\omega^{2}-k^{2}}} \hat{\mu}(k) d k \\
\left(\hat{\mathcal{D}}_{W} \hat{\mathcal{v}}\right)(\mathbf{x}) & =\frac{\operatorname{sign}\left(x-x_{0}\right)}{2} \int_{-\infty}^{\infty} e^{i k y} e^{i \sqrt{\omega^{2}-k^{2}}\left|x-x_{0}\right|} \hat{v}(k) d k \\
& =-\frac{\partial}{\partial x}\left(\hat{\mathcal{S}}_{W} \hat{v}\right)(\mathbf{x}) .
\end{aligned}
$$

Here $\hat{\mu}$ and $\hat{v}$ are the Fourier transforms of the single- and double-layer densities $\mu$ and $v$ respectively, and $x_{0}=-d / 2$ or $d / 2$ for $W=L$ or $R$ respectively. Equations (3.17)-(3.18) satisfy the usual jump relations on the wall.

From now on we will use $\hat{\xi}:=[\hat{v},-\hat{\mu}]$ as our unknown auxiliary densities, and furthermore switch to using the Fourier transform of the discrepancy functions $\left[\hat{f}, \hat{f}^{\prime}\right]$ to enforce the second block row of our linear system. The obstacle densities and mismatch remain as before. Thus (3.7) becomes,

$$
\hat{E}\left[\begin{array}{l}
\eta \\
\hat{\xi}
\end{array}\right]:=\left[\begin{array}{ll}
A & \hat{B} \\
\hat{C} & \hat{Q}
\end{array}\right]\left[\begin{array}{l}
\eta \\
\hat{\xi}
\end{array}\right]=\left[\begin{array}{l}
b \\
0
\end{array}\right]
$$

where $A$ is as before. $\hat{B}$ is the analog of $B$ with (3.17)-(3.18) in place of (2.5)-(2.6). By analogy with (3.8)-(3.9) let $\hat{S}_{V, W}$ and $\hat{D}_{V, W}$ denote (3.17) and (3.18) evaluated on a target curve $V$. Their analytic derivatives in the normal direction at the target are also easy to evaluate, analogous to (3.10)-(3.11),

$$
\begin{aligned}
\left(\hat{D}_{V, W}^{*} \hat{\mu}\right)(\mathbf{x})= & \frac{-1}{2} \int_{-\infty}^{\infty} e^{i k y} e^{i \sqrt{\omega^{2}-k^{2}}\left|x-x_{0}\right|}\left(\operatorname{sign}\left(x-x_{0}\right), \frac{k}{\sqrt{\omega^{2}-k^{2}}}\right) \\
& \cdot \mathbf{n}(\mathbf{x}) \hat{\mu}(k) d k, \quad \mathbf{x} \in V \\
\left(\hat{T}_{V, W} \hat{v}\right)(\mathbf{x})= & \frac{i}{2} \int_{-\infty}^{\infty} e^{i k y} e^{i \sqrt{\omega^{2}-k^{2}}\left|x-x_{0}\right|} \\
& \times\left(\sqrt{\omega^{2}-k^{2}}, k \operatorname{sign}\left(x-x_{0}\right)\right) \cdot \mathbf{n}(\mathbf{x}) \hat{v}(k) d k, \quad \mathbf{x} \in V .
\end{aligned}
$$

With this notation, $\hat{B}$ is given by (3.12) but with $\wedge$ applied to each entry.

Conversely, it is easy get via (3.16) the Fourier transform of the field (or its normal derivative) on the wall $W$ due to densities on $\partial \Omega$ :

$$
\left(S_{W, \partial \Omega}^{\wedge} \sigma\right)(k)=\frac{i}{4 \pi} \int_{\partial \Omega} e^{-i k y} \frac{e^{i \sqrt{\omega^{2}-k^{2}}\left|x-x_{0}\right|}}{\sqrt{\omega^{2}-k^{2}}} \sigma(\mathbf{y}) d s_{\mathbf{y}}, \quad \mathbf{y}=(x, y),
$$



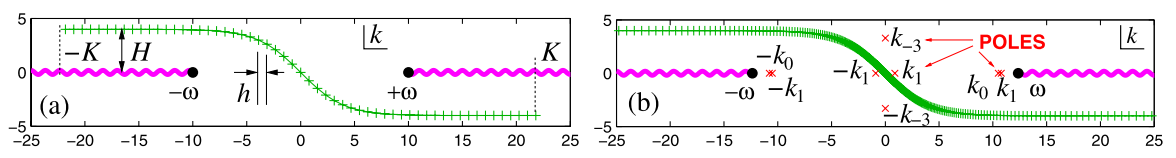

Fig. 3 (Color online) (a) Sommerfeld contour in the complex $k$ (vertical wavenumber) plane, using basic quadrature scheme (3.21)-(3.22) on a tanh curve. The pink navy lines denote branch cuts towards infinity of $1 / \sqrt{\omega^{2}-k^{2}}$. (b) Poles at the Rayleigh-Bloch vertical wavenumbers $\pm k_{n}$, and sinh-bunched nodes (3.25) giving improved convergence

$$
\begin{aligned}
\left(D_{W, \partial \Omega}^{\wedge} \tau\right)(k)= & \frac{1}{4 \pi} \int_{\partial \Omega} e^{-i k y} e^{i \sqrt{\omega^{2}-k^{2}}\left|x-x_{0}\right|} \\
& \times\left(-\operatorname{sign}\left(x-x_{0}\right), \frac{k}{\sqrt{\omega^{2}-k^{2}}}\right) \cdot \mathbf{n}(\mathbf{y}) \tau(\mathbf{y}) d s_{\mathbf{y}} \\
\left(D_{W, \partial \Omega}^{*, \wedge} \sigma\right)(k)= & \frac{1}{4 \pi} \int_{\partial \Omega} e^{-i k y} e^{i \sqrt{\omega^{2}-k^{2}}\left|x-x_{0}\right|} \operatorname{sign}\left(x-x_{0}\right) \sigma(\mathbf{y}) d s_{\mathbf{y}}, \\
\left(T_{W, \partial \Omega}^{\wedge} \tau\right)(k)= & \frac{i}{4 \pi} \int_{\partial \Omega} e^{-i k y} e^{i \sqrt{\omega^{2}-k^{2}}\left|x-x_{0}\right|} \\
& \times\left(\sqrt{\omega^{2}-k^{2}},-k \operatorname{sign}\left(x-x_{0}\right)\right) \cdot \mathbf{n}(\mathbf{y}) \tau(\mathbf{y}) d s_{\mathbf{y}} .
\end{aligned}
$$

Then $\hat{C}$ is given by (3.13) but with $(\cdot)^{\wedge}$ applied to each entry.

Finally, $\hat{Q}$ maps from Fourier to Fourier variables. Since each of the $2 \times 2$ blocks in $Q$ is a convolution, the corresponding blocks in $\hat{Q}$ are simply multiplication operators. By evaluating (3.17)-(3.18) between $L$ and $R$, and using (3.15), (3.14) becomes

$$
\begin{aligned}
\hat{Q}= & {\left[\begin{array}{ll}
I & 0 \\
0 & I
\end{array}\right] } \\
& +\frac{e^{i \sqrt{\omega^{2}-k^{2}} d}}{2}\left[\begin{array}{cc}
i\left(\alpha-\alpha^{-1}\right) / \sqrt{\omega^{2}-k^{2}} & -\alpha-\alpha^{-1} \\
\alpha+\alpha^{-1} & i\left(\alpha-\alpha^{-1}\right) \sqrt{\omega^{2}-k^{2}}
\end{array}\right],
\end{aligned}
$$

where the block is to be understood as multiplication operator in the vertical wavenumber variable $k$.

We now have explicit formulae for all blocks of the linear operator system (3.19) in which Fourier variables have been used to represent the spatially-unbounded $y$ coordinate. We will proceed to truncate this in the $k$ wavenumber domain and numerically approximate its solution.

\subsection{Quadrature of the Sommerfeld contour integral}

We now discretize (3.19) using the Nyström method; for the densities on $\partial \Omega$ this is done as before in Sect. 2. However, the domain of the Fourier variable $k$ is unbounded, and the integrand has singularities, demanding a more specialized quadrature. Equation (3.16) serves as a model for the type of integrands we need to handle; we expect this since, if our method is successful, the Fourier layer potentials on $L$ and $R$ must 
represent the effect of sources of the form (3.16) lying on neighboring copies of the obstacles - more precisely, those labelled ..., $P-2, P-1$ and $P+1, P+2, \ldots$ A complicating fact is that the infinite nature of the obstacle array sum produces Rayleigh-Bloch waves of the form (1.6)-(1.7), each wave of which introduces poles in the integrand at $\pm k_{n}$; we postpone addressing this aspect until Sect. 5. Here we present a scheme that we have found to be excellent for generic scattering parameters (i.e., not close to a Wood's anomaly).

The Sommerfeld integral (3.16) could be performed accurately on the real $k$ axis using the trapezoid rule with endpoint corrections [1] appropriate for the $(-1 / 2)$ power singularities at $\pm \omega$. However, for reasons of superior performance, of the additional poles mentioned above, and of simplicity, we instead prefer the unit-slope hyperbolic tangent contour

$$
k(\gamma)=\gamma-i H \tanh (\gamma / H), \quad \gamma \in(-\infty, \infty)
$$

shown in Fig. 3. $H$ controls the size of the curve in the $k$ plane; for unit period and obstacles of radius no larger than 2, we have found that $H=4$ is a good choice for a wide range of $\omega$. Our basic quadrature scheme is the $M$-point periodic trapezoid rule on the interval $-K \leq \gamma \leq K$, that is,

$$
\gamma_{j}=-K+2 K j / M, \quad j=1, \ldots, M,
$$

which we will see is exponentially convergent in $M$ and $K$. The quadrature approximation is $\int_{-\infty}^{\infty} g(k) d k \approx \sum_{j=1}^{M} w_{j} g\left(k_{j}\right)$ where $k_{j}=k\left(\gamma_{j}\right)$ and the weights are

$$
w_{j}=\frac{2 K}{M} k^{\prime}\left(\gamma_{j}\right)=h\left(1-i \operatorname{sech}^{2} \frac{\gamma_{j}}{H}\right) \text {. }
$$

When $|x|$ in the integrand (3.16) has a lower bound $x_{\min }$-which we expect due to the horizontal separation of the unit cell walls from the nearest obstacle images- the integrand has exponential tails. We get an estimate of a sufficient $K$ by equating the factor $e^{i \sqrt{\omega^{2}-K^{2}} x_{\min }}$ with machine precision $\varepsilon_{\text {mach }} \approx 2 \times 10^{-16}$, giving

$$
K=\sqrt{\left(\frac{\log \varepsilon_{\text {mach }}}{x_{\min }}\right)^{2}+\omega^{2}} .
$$

In practice this works well; for example, approximating our model problem (3.16) for $\omega=10$ at the point $x=x_{\min }=1, y=2$ yields $K=38$, and gives a relative error $5 \times 10^{-11}$ with $M=123$ nodes, which improves to $3 \times 10^{-15}$ with $M=179$. (In comparison, to achieve $7 \times 10^{-11}$ error using endpoint corrections on the real axis, $M=138$ is needed, and errors for larger $M$ always exceed $2 \times 10^{-14}$.) The extremely rapid convergence in $M$ just observed is actually geometric for $2 K$-periodic analytic integrands (as a function of $\gamma$ ), with a constant of convergence inversely proportional to the distance from the real axis of the nearest singularity [22, Thm. 9.28]. Despite not being periodic, our integrand is exponentially small beyond $\pm K$, thus may be treated as periodic with negligible error [44, Sect. 8].

When $\omega$ is small, the singularities at $\pm \omega$ approach the $k$-plane contour while $K$ stays large, causing a terrible convergence rate (e.g. for the above case with $\omega=0.1$, 
$M=7000$ points are needed to reach an error of $10^{-14}$ ). This may be cured by bunching the nodes towards the origin as in Fig. 3(b) via the conformal transformation,

$$
\gamma(\beta)=\lambda \delta \sinh (\beta / \lambda), \quad \beta \in(-\infty, \infty)
$$

where $\delta$ controls of the width of the central uniform node region, and $\lambda$ controls the geometric expansion rate outside this region. We apply the truncated periodic trapezoid rule to $\beta$,

$$
\beta_{j}=-L+2 L j / M, \quad j=1, \ldots, M, L=\lambda \sinh ^{-1}(K / \delta \lambda),
$$

where $L$ was chosen so that $\gamma( \pm L)= \pm K$. Back in the $k$ plane this gives nodes and (via the chain rule) weights

$$
k_{j}=k\left(\gamma\left(\beta_{j}\right)\right), \quad w_{j}=\frac{2 L}{M} \gamma^{\prime}\left(\beta_{j}\right) k^{\prime}\left(\gamma\left(\beta_{j}\right)\right),
$$

where $\gamma^{\prime}(\beta)=\delta \cosh (\beta / \lambda)$. We have not yet fully analyzed the best choice of $\delta$ and $\lambda$, but find good performance in the range $10^{-15} \leq \omega \leq 10^{3}$ with $\delta=\min (\omega / 4,1)$ and $\lambda=\max \left((\omega / 2)^{1.2}, 2\right)$, with not much sensitivity to the particular constants chosen here. For extreme frequencies, the $M$ needed for good accuracy grows: $M=$ $O(\log (1 / \omega))$ as $\omega \rightarrow 0$, and $M=O(\omega)$ as $\omega \rightarrow \infty$ (due to the growth of $K=O(\omega)$ ). For instance, returning to $\omega=0.1$, an error of $10^{-15}$ is reached at $M=177$, and there is even improvement at $\omega=10$ to an error of $4 \times 10^{-15}$ at $M=137$. This idea of transformations to improve periodic trapezoid rule convergence is not new (for a recent application and review of the literature, see Hale et al. [15]).

In summary, the Nyström method is used to approximate (3.19) by a discretized square linear system of size $2 N+2 M$,

$$
\hat{\boldsymbol{E}}\left[\begin{array}{l}
\eta \\
\xi
\end{array}\right]:=\left[\begin{array}{ll}
\boldsymbol{A} & \hat{\boldsymbol{B}} \\
\hat{\boldsymbol{C}} & \hat{\boldsymbol{Q}}
\end{array}\right]\left[\begin{array}{l}
\eta \\
\xi
\end{array}\right]=\left[\begin{array}{l}
\boldsymbol{b} \\
\mathbf{0}
\end{array}\right] .
$$

For the upper and left blocks, the $N$ quadrature nodes and weights on $\partial \Omega$ are as in Sect. 2. For the lower and right blocks we use quadrature in the Fourier variable with nodes and weights (3.25). Although the latter were justified using the model integrand (3.16), we will see in the next section that they perform very well for the full problem.

\section{Numerical examples}

We now demonstrate the performance of the basic scheme presented above, away from Wood's anomalies and when $\partial \Omega$ does not touch the walls $L$ or $R$. (Both restrictions will later be removed, in Sects. 5 and 6 respectively.) In Fig. 4(a)-(b), we show convergence with respect to $N$ (number of obstacle nodes) and $M$ (number of Fourier nodes) of the scattered field value at a single point, for a grating of simple smooth (analytic) trefoil obstacles defined in the caption, and shown in Fig. 1(a). The 

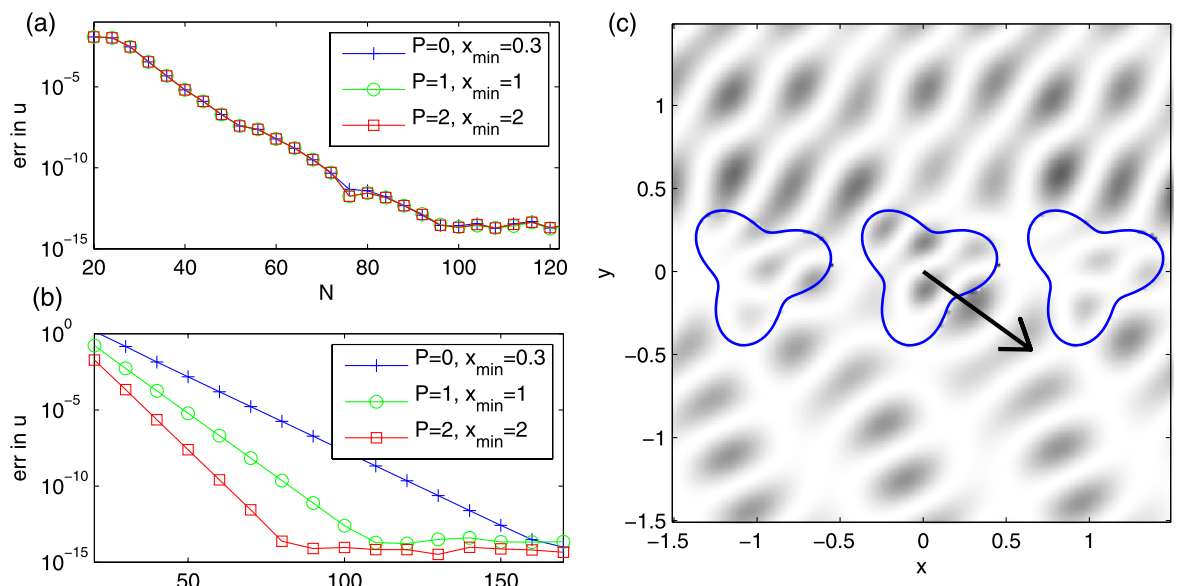

Fig. 4 Results for obstacle, index $n=1.5$, with shape given by the polar function $r(\phi)=0.35+0.105 \cos 3(\phi-\pi / 12)$ shown in Fig. 1(a), with $d=1$, and incident plane wave $\omega=10$ and $\theta=-\pi / 5$. (a) Convergence in $N$ of absolute error in field value at $(x, y)=(0.2,1)$, with $M=170$ fixed at its converged value. Three different $P$ values and $x_{\min }$ values are shown. (b) Convergence in $M$ of the same, with $N=110$ fixed at its converged value. Errors are measured relative to the converged value, being $0.19914162575818+0.24760159732486 i$ to 14 digits. (c) Total field $\left(\operatorname{Re}\left[u^{t}\right]\right)^{2}$ (i.e. electric energy density at one instant of time), plotted over three periods. Black indicates large values, white zero (dark patches are half a wavelength apart). The arrow shows incident $\theta$

convergence is exponential in both parameters. Increasing $P$ includes more directlysummed neighbors in the $A$ block of the system, hence the fields due to these neighbors no longer have to be represented by the Fourier layer potentials. Thus $x_{\min }$ can be increased, which increases the convergence rate in $M$, as shown in (b). (Changing $P$ has no effect on convergence in $N$, as (a) shows). Why then should the user not increase $P$ without limit? The answer is that computing the extra direct sums required to fill the matrix elements in the $A$ block, and evaluate the solution field, takes more time. The optimal $P$ will depend on the trade-off between matrix filling plus field evaluation vs linear system solution time.

With $P=2, x_{\min }=2$, at parameters $N=110, M=90$, at which 14 digits of the field value have converged, the CPU time required ${ }^{3}$ to fill the matrix is $0.5 \mathrm{sec}$, and $0.1 \mathrm{sec}$ to solve the complex dense linear system of size $2 N+2 M=400$. The scattered field is evaluated, using the discretized version of (3.6), on a grid ( $x$ - and $y$-spacing 0.02, with 7500 grid points) in $6 \mathrm{sec}$, giving Fig. 4(c). We note that, in contrast to the method of [7], there are no issues with evaluation of the Fourier layer potentials close to the walls: $\operatorname{dist}(\partial \Omega, R)$ is only 0.056 . The quadrature scheme is accurate up to the boundary of the unit cell, as long as $|y|$ is not too large (with these parameters, less than about 2). However, as usual with boundary integral equations,

\footnotetext{
${ }^{3}$ Here, and elsewhere in this work, computation times are for a 2006-era laptop with $2 \mathrm{GHz}$ Intel Core Duo CPU and 2 GB of RAM, running MATLAB 2008a and MPSpack (see Appendix). There is also significant overhead in the object-oriented code.
} 
full-accuracy evaluation up to the boundary of the obstacle would require specialized methods.

The linear system $(3.26)$ has $\operatorname{cond}(\hat{\boldsymbol{E}})=9 \times 10^{4}$. If we perform simple left and right diagonal preconditioning of the system matrix $\hat{\boldsymbol{E}}$ - first scaling rows to have unit 2-norm, then scaling columns to have unit 2-norm-we get a new system with matrix denoted by $\hat{\boldsymbol{E}}_{s}$ which has a reduced condition number of $4 \times 10^{2}$. This is only a little larger than for the isolated-obstacle problem of Sect. 2, which here has $\operatorname{cond}\left(\boldsymbol{A}_{0}\right)=31$.

What happens in this example if the frequency is changed? At the low frequency $\omega=0.1,14$ digit accuracy is easily reached by increasing $M$ to 180 . As $\omega \rightarrow 0$, we observe cond $\left(\hat{\boldsymbol{E}}_{S}\right)=O(1 / \omega)$ but note that, since $u=O(\omega)$, an absolute error of $10^{-14}$ is still achieved. At the high frequency $\omega=50$ ( 8 wavelengths per period), one needs $N=230$, but only $M=160$, to achieve errors around $10^{-14}$; solution time is $2 \mathrm{sec}$ to fill and $0.6 \mathrm{sec}$ to solve, and cond $\left(\hat{\boldsymbol{E}}_{S}\right)$ is only $7 \times 10^{2}$.

\subsection{Extracting Bragg amplitudes and checking flux conservation}

In applications it is crucial to know the far-field behavior, i.e., the amplitudes of the propagating Bragg orders $c_{n}$ in (1.6) and $d_{n}$ in (1.7). We compute these from the density vector $[\boldsymbol{\eta} ; \boldsymbol{\xi}]$, by using (3.6) to evaluate $u$ and $u_{n}$ on the horizontal lines $-d / 2<x<d / 2, y= \pm y_{0}$. (We choose $y_{0}$ to leave at least $d / 2$ separation from $\partial \Omega$.) On the bottom side, for each mode $n$ for which $k_{n} \neq 0$,

$$
\left[\begin{array}{l}
d_{n} \\
b_{n}
\end{array}\right]=\frac{1}{2}\left[\begin{array}{cc}
1 & -1 / i k_{n} \\
1 & 1 / i k_{n}
\end{array}\right]\left[\begin{array}{c}
d^{-1} \int_{-d / 2}^{d / 2} u\left(x,-y_{0}\right) e^{-i \kappa_{n} x} d x \\
d^{-1} \int_{-d / 2}^{d / 2} u_{y}\left(x,-y_{0}\right) e^{-i \kappa_{n} x} d x
\end{array}\right],
$$

where the integrals extract Fourier series coefficients of the $d$-periodic field $u\left(x,-y_{0}\right) e^{-i \alpha x / d}$ and its $y$-derivative. The $2 \times 2$ matrix projects this into downwards $\left(d_{n}\right)$ and upwards $\left(b_{n}\right)$ propagating amplitudes. Similarly, replacing $-y_{0}$ by $y_{0}$ in (4.1) gives the pair $\left[a_{n} ; c_{n}\right]$, namely the downwards and upwards amplitudes on the top side (see Fig. 1(a)). Neither "incoming" amplitude $a_{n}$ nor $b_{n}$ is needed here-they are zero for a scattered field obeying the radiation conditions-but one will rear its head in Sect. 5. We approximate the integrals numerically via 20-point Gaussian quadrature.

An independent test of the accuracy of the method is then given by the conservation of (energy) flux. For the exact full field it holds that total outgoing flux equals total incoming flux [27, Eq. (40)], [40, p. 12], that is,

$$
\sum_{k_{n}>0} k_{n}\left(\left|c_{n}\right|^{2}+\left|d_{n}+\delta_{n, 0} e^{-i k^{\mathrm{i}} y_{0}}\right|^{2}\right)=k^{\mathrm{i}}
$$

where the term with the Kronecker delta accounts for the unit-amplitude incident plane wave in the $n=0$ mode. In the above $\omega=10$ example the "flux error" (difference between left and right hand sides of the above) is $3 \times 10^{-14}$, consistent with the error estimated from convergence of $u$ at a point; results are similar at the other $\omega$ values. 


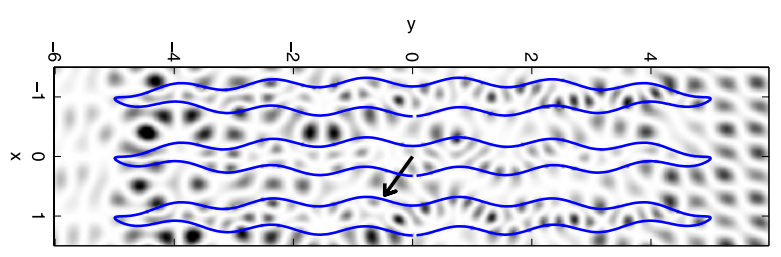

Fig. 5 High aspect ratio obstacle: a tall wobbly ellipse given in Cartesians by $(0.25 \cos \phi+0.075 \cos (5 \sin \phi), 0.25 \sin \phi), 0 \leq \phi<2 \pi$, with incident plane wave $\omega=10$ and $\theta=-\pi / 5$ (shown by arrow). Total field $\left(\operatorname{Re}\left[u^{\mathrm{t}}\right]\right)^{2}$ is plotted over three periods. The figure has been rotated by $-\pi / 2$ to fit on the page

Table 1 Results for scattering from the wobbly ellipse grating of height 10 periods. "Conv. error" is the approximate error estimated from convergence of a field value $u^{\mathrm{t}}(0.2,5.5)$

\begin{tabular}{lllllllll}
\hline$\omega$ & $N$ & $M$ & Flux error & Conv. error & $t_{\text {fill }}$ & $t_{\text {solve }}$ & $\operatorname{cond}(\hat{\boldsymbol{E}})$ & $\operatorname{cond}\left(\hat{\boldsymbol{E}}_{s}\right)$ \\
\hline 10 & 500 & 330 & $3 \mathrm{e}-13$ & $1 \mathrm{e}-13$ & $9.5 \mathrm{sec}$ & $4.5 \mathrm{sec}$ & $6 \mathrm{e} 6$ & $1.6 \mathrm{e} 4$ \\
20 & 900 & 450 & $2 \mathrm{e}-12$ & $1 \mathrm{e}-12$ & $29 \mathrm{sec}$ & $18 \mathrm{sec}$ & $1.6 \mathrm{e} 8$ & $1.3 \mathrm{e} 5$ \\
\hline
\end{tabular}

\subsection{High aspect ratio example}

One motivation for our work is handling high aspect ratio obstacles. Here we present such an example. For tall obstacles many periods in height where $y_{\max }:=$ $\max _{(x, y) \in \partial \Omega}|y| \gg 1$ (we assume $d=1$ ), exponential growth of the factor $e^{i k y}$ in the imaginary $k$ direction causes undesirably large integrands along the Sommerfeld contour, hence large matrix entries and large condition number. This leads us to reduce the scale of the tanh curve (3.21); we choose the height $H=\min \left(4,3 / y_{\max }\right)$. A sideeffect is that a larger $M$ is needed since the contour comes closer to the singularities on the real axis. Thus the choice of $H$ is a trade-off between matrix size and condition number.

In Fig. 5 we show the full field for the distorted ellipse of height 10 periods described in the caption, at $\omega=10$, computed using $P=2, x_{\min }=2.5$, and $\delta=\omega / 4$. Table 1 gives numerical parameters for this case, and also for higher wavenumber (at $\omega=20$ the scatterer is 32 exterior wavelengths long, and 48 interior wavelengths). $t_{\text {fill }}$ is the time required to fill the matrix $\hat{\boldsymbol{E}}$, and $t_{\text {solve }}$ the time to solve the dense linear system of size $2 N+2 M$. In both cases, the $N$ required is almost identical to the smallest $N$ needed to achieve similar accuracy in the isolated obstacle scattering problem (Sect. 2). The plot in Fig. 5 took 24 sec to evaluate (grid spacing 0.04, with 7500 points).

It is clear that condition numbers appear to be growing with $\omega$ (this is also true for the isolated obstacle, but to a lesser extent). As mentioned above, they can be lowered, by about an order of magnitude, by decreasing $H$; however, this requires a somewhat larger $M$. The values shown are a good compromise that achieves 12-digit accuracy. 


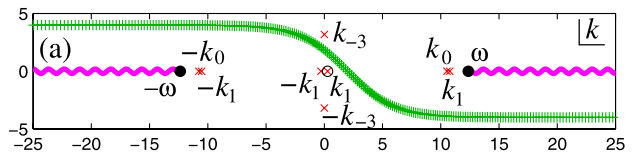

(c) 1
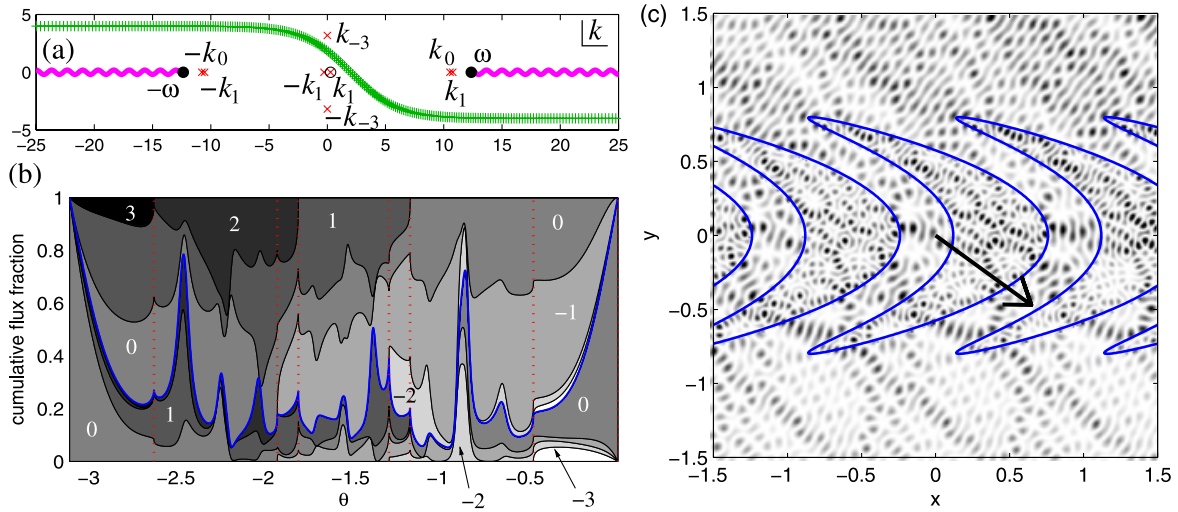

Fig. 6 (Color online) (a) Deformed (translated) Sommerfeld contour; compare Fig. 3(b). The contour has crossed the circled pole. (b) Outgoing flux fractions for all Bragg modes $(-3 \leq n \leq 3$, some labelled by $n$ ) for the example of Fig. 4, as a function of incident angle $\theta \in(-\pi, 0)$. The top group (above blue line) are downwards (transmitted), the bottom group upwards (reflected). Note the square-root type cusps at Wood's anomalies (shown by vertical dotted lines). (c) Total field $\left(\operatorname{Re}\left[u^{\mathrm{t}}\right]\right)^{2}$ for kite-shaped obstacles of index $n=1.5$ and shape $(0.32 \cos \phi+0.64 \cos 2 \phi-0.2,0.8 \sin \phi)$, which wrap around the unit cell; see Sect. 6

\section{Scattering parameters near to and at Wood's anomalies}

So far we have assumed that we are not close to or at a Wood's anomaly, i.e. that no $k_{n}$ is close to the origin. Let us take a moment to explain why, in the representation of a scattered solution, there is generically a pole in the integrand (i.e. Fourier densities $\hat{\mu}(k)$ and $\hat{v}(k)$ in (3.17)-(3.18)) at $k= \pm k_{n}$, for each $n$ (both propagating and evanescent); see Fig. 3(b). Distributionally, we have the following Fourier transform pair, for $k_{p} \in \mathbb{C}$,

$$
\begin{aligned}
& \hat{g}_{p}(k)=\frac{1}{2 \pi i\left(k-k_{p}\right)}, \\
& g_{p}(y)=\left\{\begin{array} { l l } 
{ e ^ { i k _ { p } y } , } & { y > 0 , } \\
{ 0 , } & { y < 0 , }
\end{array} \text { or } \quad \left\{\begin{array}{ll}
0, & y>0, \\
-e^{i k_{p} y}, & y<0,
\end{array}\right.\right.
\end{aligned}
$$

where in the first (second) case the pole $k_{p}$ lies above (below) the contour.

(This is easily checked using Jordan's lemma and the residue theorem.) Since both Rayleigh-Bloch expansions (1.6) and (1.7) — thus the densities that produce themare represented by the same $y$-direction Fourier function, this function must have a pole at each $k_{n}$ in order to capture the outgoing amplitude for $y>0$ but zero incoming amplitude for $y<0$. This pole must lie above the Sommerfeld contour to generate this first case rather than the second. By a similar argument, there are poles at $-k_{n}$, which must lie below the contour.

The astute reader will have noticed that these poles $\pm k_{n}$ are closer to the Sommerfeld contour (3.21) than are $\pm \omega$, thus realize that they (rather than $\omega$ ) should control the node bunching parameter $\delta$ in (3.24). We have tested this idea, choosing e.g. $\delta=\min \left(\left|k_{n}\right|\right) / 2$; however matrix condition number appears to grow as $O\left(1 /\left|k_{n}\right|\right)$, 
losing digits of accuracy - in fact at the same rate as would a conventional quasiperiodic Green's function based method. This approach also requires large $M$ and fails to get more than a few correct digits when $k_{n}=0$.

Our proposal is to translate the Sommerfeld contour by $O(1)$ along the positive real axis, so as to move it to be a distance at least 1 away from the trouble at the origin (and in fact from any other $k_{n}$ pole; see Fig. 6(a)). In doing so, the contour crosses a pole, which we label by $n=p$. From the above discussion, this corresponds to solving for a field $u$ with peculiar radiation conditions: the $p$ th mode is incoming from below $\left(c_{p}=a_{p}=0\right)$, instead of the usual outgoing from above $\left(b_{p}=a_{p}=0\right)$, whilst all other modes $n \neq p$ are as usual. To fix this we add to (3.6), the representation of $u$ in $U \backslash \bar{\Omega}$, the quasi-periodic plane wave $a e^{i \kappa_{p} x} e^{i k_{p} y}$, where $a$ is an additional unknown. This accounts for the difference between the two cases in (5.1). An additional constraint is then needed: we impose the correct mode incoming radiation condition $b_{p}=0$. This adds to the linear system (3.26) a row whose entries are filled using quadrature applied to the expression for $b_{p}$ in (4.1), giving,

$$
\left[\begin{array}{ccc}
\boldsymbol{A} & \hat{\boldsymbol{B}} & \boldsymbol{a}_{p} \\
\hat{\boldsymbol{C}} & \hat{\boldsymbol{Q}} & \mathbf{0} \\
\boldsymbol{r}_{p} & \boldsymbol{s}_{p} & e^{-i k_{p} y_{0}}
\end{array}\right]\left[\begin{array}{l}
\boldsymbol{\eta} \\
\boldsymbol{\xi} \\
a
\end{array}\right]=\left[\begin{array}{l}
\boldsymbol{b} \\
\mathbf{0} \\
0
\end{array}\right],
$$

a square system of size $2 N+2 M+1$. The column vector $\boldsymbol{a}_{p}$ expresses the effect of the new plane wave on the obstacle mismatch $\left[u^{+}-u^{-} ; u_{n}^{+}-u_{n}^{-}\right]$at the nodes on $\partial \Omega$.

We apply the above whenever $\left|k_{n}\right|<1$ for some $n$, and when this occurs for two values of $n$ (close to a double Wood's anomaly), we apply its natural generalization to two extra degrees of freedom. Precisely at a Wood's anomaly, $k_{n}=0$ and the projection in (4.1) onto exponentials degenerates into a projection onto the functions $\{1, y\}$, of which only the constant function is a valid radiation condition, thus we replace the $2 \times 2$ matrix in this formula by the identity. Since the contour is now far from any pole, we might hope that the convergence in $M$ while close to or at a Wood's anomaly is as good as it was far from one. This is true: choosing the example of Fig. 4, with $\omega=10$, and $\theta=\cos ^{-1}(1-2 \pi / \omega d)$ which is a single Wood's anomaly for mode $n=1$, we achieve convergence at $N=110, M=90$ to 14 digit accuracy to the value $u^{\mathrm{t}}(0.2,1)=-0.61990437785568+0.46197831200416 i$. Condition number after left and right diagonal preconditioning is $3.4 \times 10^{3}$, an order of magnitude larger than before, but still very reasonable.

We thus have made our scheme immune to Wood's anomalies. This lets us produce Fig. 6(b) which shows the flux fractions (individual terms in (4.2) normalized to unit total) in each of the modes, swept over all incident angles $-\pi<\theta<0$. The idea of adding extra discrete unknowns and conditions to an integral equation system dates back at least to Mikhlin [29, §31]. A similar idea has been recently used for an array of sound-soft or sound-hard discs, to 2 significant digits [27]. Removal of singularities near a contour to improve convergence rates has also been used to evaluate periodic Green's functions [17]. 


\section{Obstacle intersecting unit cell walls}

When $\partial \Omega$ intersects $L$ or $R$, the method presented so far fails, crudely speaking because parts of the obstacle see the fields on the wrong side of the wall layer potential. However, we can fix the scheme to handle this case in the following simple way. We redefine $L$ to be the vertical line at location $x_{0}=-(1 / 2+Z) d$, and $R$ at location $x_{0}=(1 / 2+Z) d$, with $Z=0,1, \ldots$ a new method parameter. Discrepancies (3.1)-(3.2) are redefined using $\alpha^{2 Z+1}$ as the phase instead of $\alpha$, and thus measure the difference from quasi-periodicity across a strip $2 Z+1$ periods wide. We use the representation (3.6), again replacing $\alpha$ by $\alpha^{2 Z+1}$. We ensure $P \geq Z$ so that obstacle images fill this wider strip. To construct the blocks of the linear system (3.7), we set the mismatch on $\partial \Omega$ as before, i.e. equal to $b$ as defined in (2.9), and impose vanishing discrepancy, as before. Note that $Z=0$ corresponds to the original method presented in Sect. 3.

Conjecture 2 The method described above for $Z>0$ has the same solution field $u$ in the strip $(-d / 2, d / 2) \times(-\infty, \infty)$ as the method with $Z=0$.

This is perhaps surprising since the mismatch is only enforced on one of the many copies of $\partial \Omega$ which fall within the wider strip. Pending a formal proof, we explain this (away from Wood's anomalies) as follows. Using the Schur complement of the upper-left block in (3.19) we have

$$
A_{\mathrm{QP}} \eta=b \quad \text { where } A_{\mathrm{QP}}:=A-\hat{B}(\hat{Q})^{-1} \hat{C} .
$$

As in [7], it is easy to check that $A_{\mathrm{QP}}$ is now $A_{0}$ of (2.8) but with quasi-periodic Green's functions ((3.4)-(3.5) with $P=\infty)$ in the exterior. This argument applies for any $Z=0,1, \ldots$, thus the solution $\eta$, hence the full field, is independent of $Z$.

Implementing this gives the following changes in the operator system (3.7) and hence (3.19): The phase $\alpha$ becomes $\alpha^{2 Z+1}$ in $B$ and $Q$, i.e. (3.12), (3.14) and (3.20). Note that $B$ now involves larger interaction distances of order $Z d$. For the $C$ block, less cancellation occurs, as shown in Fig. 2(c)-(d), generalizing (3.13) to

$$
C=\left(\sum_{j=P-2 Z}^{P}-\sum_{j=-P-1}^{-P-1+2 Z}\right) \alpha^{j}\left[\begin{array}{ll}
D_{L-j \mathbf{d}, \partial \Omega} & -S_{L-j \mathbf{d}, \partial \Omega} \\
T_{L-j \mathbf{d}, \partial \Omega} & -D_{L-j \mathbf{d}, \partial \Omega}^{*}
\end{array}\right]
$$

$Z=1$ is sufficient for wall-intersecting obstacles that are not much wider than $d$. It was used, for example, in Fig. 6(c), where we compute diffraction from a grating of kite-shaped obstacles which necessarily intersect any artificial vertical unit cell walls. The frequency $\omega=50$ is quite high, needing $N=550$, but only $M=170$, to achieve convergence error of around $10^{-13}$ and flux error $4 \times 10^{-14}$. Computation times are $11.5 \mathrm{sec}$ to fill, $3 \mathrm{sec}$ to solve, and $170 \mathrm{sec}$ to evaluate $u$ at 30000 points for the plot, and $\operatorname{cond}\left(\hat{\boldsymbol{E}}_{S}\right)$ is $8.8 \times 10^{3}$.

In summary, by widening the scheme to one which quasi-periodizes across more than one period, we have effectively made the artificial walls "invisible" to the obstacle, without increasing the computational effort. 


\section{Conclusions}

We have quasi-periodized a scheme for dielectric obstacle scattering, converting it into a scheme for an infinite grating of such obstacles, using only the free-space Green's function and auxiliary layer potentials based on Sommerfeld integrals on the unit cell walls. For typical frequencies $\omega d / 2 \pi=O(1)$, independently of the complexity of the obstacle, this adds only around 200 extra unknowns to the linear system, keeps condition number reasonable (the scheme is 2 nd kind), and achieves errors close to machine precision, uniformly in incident angle.

As far as we are aware, this is the first time an integral equation approach to this problem has been formulated that works at Wood's anomalies (for surfaces see [2]). This is a promising alternative to the use of quasi-periodic Green's functions, which fail around Wood's anomalies, can be complicated to evaluate, and are complicated to use in high aspect ratio cells when based on lattice sums. We expect this advantage to be more pronounced for doubly-periodic problems in 3D, and when the obstacle scattering is accelerated by fast-multipole methods (FMM).

Some issues remain open for study, such as: (i) ill-conditioning for very low $\omega$ and high aspect ratios, (ii) better automated choice of method parameters $H, \lambda, \delta, P$ and $Z$, and (iii) a proof of Conjecture 2 for all scattering parameters. There are several acceleration ideas that we have not presented here. Since $\hat{\boldsymbol{E}}$ is a matrix polynomial in $\alpha$, the coefficients can be pre-computed and used to fill the matrix quickly for multiple incident angles. We have only presented results using dense linear algebra (not even using the fact that $\hat{Q}$ is diagonal), so assessing GMRES convergence in large-scale examples with FMM acceleration is a next step.

We also note that the discretized version of the Schur complement (6.1) seems like a good $O\left(N^{2} M\right)$ way to compute $N^{2}$ evaluations of the quasi-periodic Green's function, should they be required in a more conventional solver. In the case of Fig. 4, for example, this allows each entry to be filled in around $10^{-5} \mathrm{sec}$, which is competitive with the $10^{-4}$ to $10^{-3} \mathrm{sec}$ recently reported [3].

The algorithm we present is available through a free toolbox for MATLAB, as shown in the Appendix. Barring a few subtleties, we expect the method to generalize to multi-layered media with periodic inclusions, opening up a wealth of technological applications; we will study this in a future paper.

Acknowledgements We thank Stephen Shipman and Simon Chandler-Wilde for invaluable discussions. The work of AHB was supported by NSF grant DMS-0811005, and by the Class of 1962 Fellowship at Dartmouth College. The work of LG was supported by the Department of Energy under contract DEFG0288ER25053 and by AFOSR under MURI grant FA9550-06-1-0337.

Open Access This article is distributed under the terms of the Creative Commons Attribution Noncommercial License which permits any noncommercial use, distribution, and reproduction in any medium, provided the original author(s) and source are credited.

\section{Appendix: Simple MATLAB code example in MPSpack}

MPSpack is an object-oriented MATLAB toolbox for solving 2D Helmholtz PDE problems recently developed by Betcke and one of the authors [6]. The methods of 
the present paper have been incorporated into version 1.1 beta of MPSpack. The package is freely available at http://code.google.com/p/mpspack. The download section of that Web page also includes an extensive tutorial and a technical manual. Below we give a short, 12-line, human-readable code which implements the methods of this paper to achieve 14 digits of accuracy in solving the quasi-periodic scattering problem of Sect. 4. The gpscatt class has been written in such a way that it builds upon the layer-potential scheme for the non-periodic scattering problem. The integer parameter $P$ is called nei, and $Z$ is called buf. Handling more complicated obstacles is quite easy; this, and other details, are explained in the tutorial.

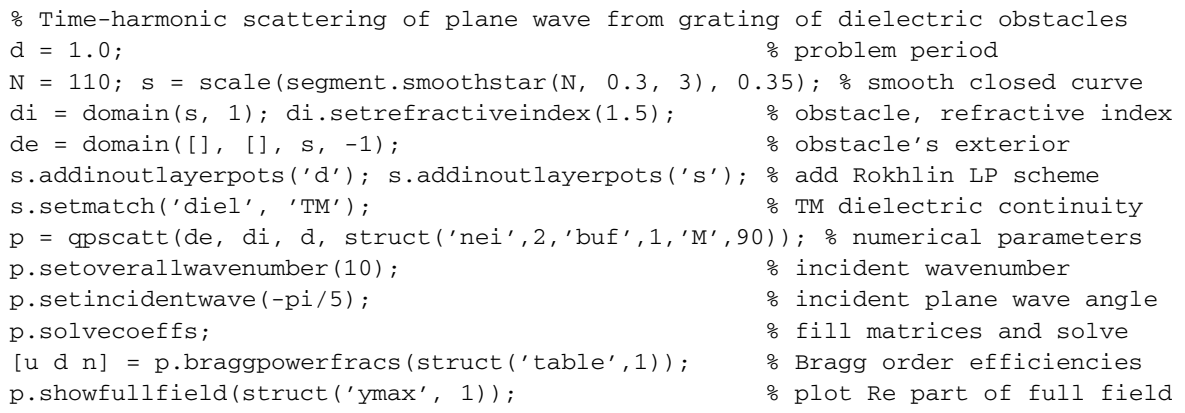

\section{References}

1. Alpert, B.K.: Hybrid Gauss-trapezoidal quadrature rules. SIAM J. Sci. Comput. 20, 1551-1584 (1999)

2. Arens, T., Chandler-Wilde, S.N., DeSanto, J.A.: On integral equation and least squares methods for scattering by diffraction gratings. Commun. Comput. Phys. 1, 1010-1042 (2006)

3. Arens, T., Sandfort, K., Schmitt, S., Lechleiter, A.: Analysing Ewald's method for the evaluation of Green's functions for periodic media. IMA J. Numer. Anal. (2010, submitted). Available at http://digbib.ubka.uni-karlsruhe.de/volltexte/1000019136

4. Atwater, H.A., Polman, A.: Plasmonics for improved photovoltaic devices. Nature Mater. 9(3), 205213 (2010)

5. Bao, G., Dobson, D.C.: Modeling and optimal design of diffractive optical structures. Surv. Math. Ind. 8, 37-62 (1998)

6. Barnett, A.H., Betcke, T.: An exponentially convergent non-polynomial finite element method for time-harmonic scattering from polygons. SIAM J. Sci. Comput. 32(3), 1417-1441 (2010)

7. Barnett, A.H., Greengard, L.: A new integral representation for quasi-periodic fields and its application to two-dimensional band structure calculations. J. Comput. Phys. 229, 6898-6914 (2010)

8. Barty, C.P.J., et al.: An overview of LLNL high-energy short-pulse technology for advanced radiography of laser fusion experiments. Nuclear Fusion 44(12), S266 (2004)

9. Bonnet-BenDhia, A.-S., Starling, F.: Guided waves by electromagnetic gratings and non-uniqueness examples for the diffraction problem. Math. Methods Appl. Sci. 17, 305-338 (1994)

10. Colton, D., Kress, R.: Integral Equation Methods in Scattering Theory. Wiley, New York (1983)

11. Colton, D., Kress, R.: Inverse Acoustic and Electromagnetic Scattering Theory. Applied Mathematical Sciences, vol. 93, 2nd edn. Springer, Berlin (1998)

12. Dienstfrey, A., Hang, F., Huang, J.: Lattice sums and the two-dimensional, periodic Green's function for the Helmholtz equation. Proc. R. Soc. Lond. A 457, 67-85 (2001)

13. Garabedian, P.R.: Partial Differential Equations. Wiley, New York (1964)

14. Guenther, R.B., Lee, J.W.: Partial Differential Equations of Mathematical Physics and Integral Equations. Prentice Hall, Englewood Cliffs (1988)

15. Hale, N., Higham, N.J., Trefethen, L.N.: Computing $A, \log (A)$, and related matrix functions by contour integrals. SIAM J. Numer. Anal. 46(5), 2505-2523 (2008) 
16. Holter, H., Steyskal, H.: Some experiences from FDTD analysis of infinite and finite multi-octave phased arrays. IEEE Trans. Antennae Propag. 50(12), 1725-1731 (2002)

17. Horoshenkov, K.V., Chandler-Wilde, S.N.: Efficient calculation of two-dimensional periodic and waveguide acoustic Green's functions. J. Acoust. Soc. Am. 111, 1610-1622 (2002)

18. Jackson, J.D.: Classical Electrodynamics, 3rd edn. Wiley, New York (1998)

19. Joannopoulos, J.D., Johnson, S.G., Meade, R.D., Winn, J.N.: Photonic Crystals: Molding the Flow of Light, 2nd edn. Princeton Univ. Press, Princeton (2008)

20. Kelzenberg, M.D., Boettcher, S.W., Petykiewicz, J.A., Turner-Evans, D.B., Putnam, M.C., Warren, E.L., Spurgeon, J.M., Briggs, R.M., Lewis, N.S., Atwater, H.A.: Enhanced absorption and carrier collection in Si wire arrays for photovoltaic applications. Nature Mater. 9(3), 239-244 (2010)

21. Kress, R.: Boundary integral equations in time-harmonic acoustic scattering. Math. Comput. Model. 15, 229-243 (1991)

22. Kress, R.: Numerical Analysis. Graduate Texts in Mathematics, vol. 181. Springer, Berlin (1998)

23. Kurkcu, H., Reitich, F.: Stable and efficient evaluation of periodized Green's functions for the Helmholtz equation at high frequencies. J. Comput. Phys. 228, 75-95 (2009)

24. Li, L., Chandezon, J., Granet, G., Plumey, J.P.: Rigorous and efficient grating-analysis method made easy for optical engineers. Appl. Opt. 38(2), 304-313 (1999)

25. Linton, C.M.: The Green's function for the two-dimensional Helmholtz equation in periodic domains. J. Eng. Math. 33, 377-402 (1998)

26. Linton, C.M.: Lattice sums for the Helmholtz equation. SIAM Rev. 52(4), 630-674 (2010). doi: $10.1137 / 09075130 \mathrm{X}$

27. Linton, C.M., Thompson, I.: Resonant effects in scattering by periodic arrays. Wave Motion $\mathbf{4 4}, 165-$ 175 (2007)

28. McPhedran, R.C., Nicorovici, N.A., Botten, L.C., Grubits, K.A.: Lattice sums for gratings and arrays. J. Math. Phys. 41, 7808-7816 (2000)

29. Mikhlin, S.G.: Integral Equations, 2nd edn. MacMillan, New York (1964)

30. Model, R., Rathsfeld, A., Gross, H., Wurm, M., Bodermann, B.: A scatterometry inverse problem in optical mask metrology. J. Phys., Conf. Ser. 135, 012,071 (2008)

31. Moroz, A.: Exponentially convergent lattice sums. Opt. Lett. 26, 1119-21 (2001)

32. Morse, P., Feshbach, H.: Methods of Theoretical Physics, vol. 1. McGraw-Hill, New York (1953)

33. Müller, C.: Foundations of the Mathematical Theory of Electromagnetic Waves. Springer, Berlin (1969)

34. Nédélec, J.C., Starling, F.: Integral equation methods in a quasi-periodic diffraction problem for the time-harmonic Maxwell's equations. SIAM J. Math. Anal. 22(6), 1679-1701 (1991)

35. Nicholas, M.J.: A higher order numerical method for 3-D doubly periodic electromagnetic scattering problems. Commun. Math. Sci. 6(3), 669-694 (2008)

36. Otani, Y., Nishimura, N.: A periodic FMM for Maxwell's equations in 3D and its applications to problems related to photonic crystals. J. Comput. Phys. 227, 4630-4652 (2008)

37. Peter, M.A., Meylan, M.H., Linton, C.M.: Water-wave scattering by a periodic array of arbitrary bodies. J. Fluid Mech. 548, 237-256 (2006)

38. Petit, R. (ed.): Electromagnetic Theory of Gratings, Topics in Current Physics, vol. 22. Springer, Heidelberg (1980)

39. Rokhlin, V.: Solution of acoustic scattering problems by means of second kind integral equations. Wave Motion 5, 257-272 (1983)

40. Shipman, S.: Resonant scattering by open periodic waveguides. In: Progress in Computational Physics (PiCP), vol. 1, pp. 7-50. Bentham Science Publishers, Dubai (2010)

41. Shipman, S., Venakides, S.: Resonance and bound states in photonic crystal slabs. SIAM J. Appl. Math. 64, 322-342 (2003)

42. Taflove, A.: Computational Electrodynamics: The Finite-Difference Time-Domain Method. Artech House, Norwood (1995)

43. Venakides, S., Haider, M.A., Papanicolaou, V.: Boundary integral calculations of two-dimensional electromagnetic scattering by photonic crystal Fabry-Perot structures. SIAM J. Appl. Math. 60, 1686$1706(2000)$

44. Weideman, J.A.C.: Numerical integration of periodic functions: a few examples. Am. Math. Mont. 109(1), 21-36 (2002) 
45. Wojcik, G.L.J.M. Jr., Marx, E., Davidson, M.P.: Numerical reference models for optical metrology simulation. In: SPIE Microlithography 92: IC Metrology, Inspection, and Process Control VI, vol. 1673-06 (1992)

46. Wood, R.W.: On a remarkable case of uneven distribution of light in a diffraction grating spectrum. Philos. Mag. 4, 396-408 (1902)

47. Zhang, B., Chandler-Wilde, S.N.: Integral equation methods for scattering by infinite rough surfaces. Math. Methods Appl. Sci. 26, 463-488 (2003) 